\title{
The Automated Test Case Design and Realization of Smart TV Operating System Based on TVOS
}

\author{
FU Rui, ZHAO LiangFu, Zhang DingJing, LI Zheng ${ }^{3, c}$, BAI Wei \\ Academy of Broadcasting Science,SAPPRFT, Beijing 100866, China \\ furui@abs.ac.cn
}

Keywords: Smart TV operating system; information safety; trusted computing; framework

\begin{abstract}
In this paper, the author analyzed the safety environment faced by the smart TV operating system and proposed the safety technology system based on trusted computing technology route, conforming to smart TV operating system TVOS layering framework and having defense in depth by combining with broadcasting business safety needs.
\end{abstract}

\section{Introduction}

In order to promote standardization and intelligence of intelligent terminals, science and technology division in State Administration of Press, Publication, Radio, Film and Television of the People's Republic of China(SARFT) launched research work of smart TV operating(TVOS) key technology with relevant scientific research institutes, enterprises and public institutions and launched TVOS1.0 version in 2013. In 2015, TVOS2.0 version was launched, symbolizing that the smart TVOS developed independently in China has gotten the staging achievements. TVOS was officially applied. TVOS safety technology is technical means and method to target at the safety threat to the smart operating system by formulating and applying the safety strategies, constructing safety model and realizing safety mechanism. TVOS safety technology system is based on the uniform trusted computing technology route and applies the open and sustainable evolvement development technology mode. It has been proposed by combining with TVOS software laying framework features and constructing and applying TVOS safety strategy and applying safety protection ability, and aiming at the testing of smart TVOS. It is the precondition and safeguard to realize standardization and normalization for TVOS software system. The timely publication of TVOS software system will meet the urgent needs of standardization and intelligence for the digital TV terminals of wired network companies in various areas. It also lays a solid foundation on the standardization and intelligence of digital TV terminals in SARFT. In order to ensure that TVOS can be rapidly and effectively deployed in the nationwide wired network, it is necessary to conduct the strict and comprehensive authentication test for standards and rules, so as to ensure normalization and standardization of TVOS software system and provide support for unifying Chinese smart TV terminals and closing the regional network division. Under the background, to carry out TVOS testing, construct the uniform and comprehensive testing environment platform, develop standard testing cases and automated testing tools, and apply scientific and high-efficient testing methods is the precondition and safeguard to realize the standardization and normalization of TVOS software system.

\section{The Automated Framework Design of Smart TV Operating System Based on TVOS}

The TVOS aims to ensure whether the system conforms to TVOS requirements by aiming at NGB TVOS, including whether core version is correct, whether modular function is perfect, whether framework is complete and reasonable, and whether functions and interfaces are comprehensive. The technical routes will further study and analyze TVOS testing model. According to the testing model, the development of the testing process, testing tools and testing cases is conformed to construct the testing platform and write a testing scheme. 


\section{A. Design Basis}

Smart TVOS module layer gets involved in more functional modules. Meanwhile, each module also contains the respective conditional input parameters, thus the configuration document mode is applied to manage switch, input parameters and result output methods of each module, so as to realize the flexible configuration for each test-module. The test-module applies the modular code compilation mode to organize TestCase. The automated operation framework confirms modular combination or TestCase in line with the analytical results of configuration documents, so as to output test result information. As a result, the automated test framework designed in this paper mainly includes case compilation module, automated operation tool, analytical module of configuration documents, and test result output. The function and realization of automated test framework defined by us is described in Table 1.

Tab.1 Functional Description of Automated Test Framework

\begin{tabular}{|c|c|l|}
\hline No. & Functions & \multicolumn{1}{|c|}{ Specific Contents } \\
\hline 1 & $\begin{array}{c}\text { Test } \\
\text { organization }\end{array}$ & $\begin{array}{l}\text { Apply module/case; cases can be scattered in different documents. Multiple } \\
\text { modules can be merged into an executable file. }\end{array}$ \\
\hline 2 & $\begin{array}{c}\text { Test } \\
\text { structure }\end{array}$ & $\begin{array}{l}\text { According to the configuration file management, multiple tests can be excepted } \\
\text { from a whole set of testing code. }\end{array}$ \\
\hline 3 & $\begin{array}{c}\text { Abnormal } \\
\text { treatment }\end{array}$ & $\begin{array}{l}\text { Give the abnormal judgment treatment by aiming at character strings, indicators } \\
\text { and operating state }\end{array}$ \\
\hline 5 & $\begin{array}{c}\text { Test } \\
\text { implemetnat } \\
\text { ion }\end{array}$ & $\begin{array}{l}\text { Apply commands to operate the executable proceudres and command assigned } \\
\text { configuration documents. }\end{array}$ \\
\hline 5 & output & Select the output mode, directly output it to the terminal or output it to the file. \\
\hline
\end{tabular}

This automated test framework provides a kind of module and automated operation environment to compile modular test cases, so as to greatly simplify compilation process of modular test cases, show the favorable expansibility and stability, while improving development efficiency of testing products.

B. Introduction to the Development Environment

(1) Software environment: Based on the smart TVOS2.0

(2) Hardware environment: integrate with smart TV and STB of smart TVOS2.0

(3) Development programming language: $\mathrm{C} / \mathrm{C}++/$ Java

C. Software Framework Design

The automated testing software framework of the smart TVOS modules is shown in Figure 1, including TVOS core layer, TVOS modular layer, TestCase layer, and test framework layer, showing the functions of each layer in the test system and position in the systematic framework. (1) TVOS core layer includes the bottom layer protocol and driver matched with the hardware; (2) TVOS modular layer includes digital TV module, media disposal module, man-machine interaction module, and audio/video setting module. (3) TestCase layer is the test case set of corresponding modules in each functional module of TVOS modular layer. (4) Test framework layer contains the basic module of test cases, automated operation framework, analytical management of configuration documents and output module of test results, showing the favorable expansibility and stability. It can flexibly control operational environment and conditions. 

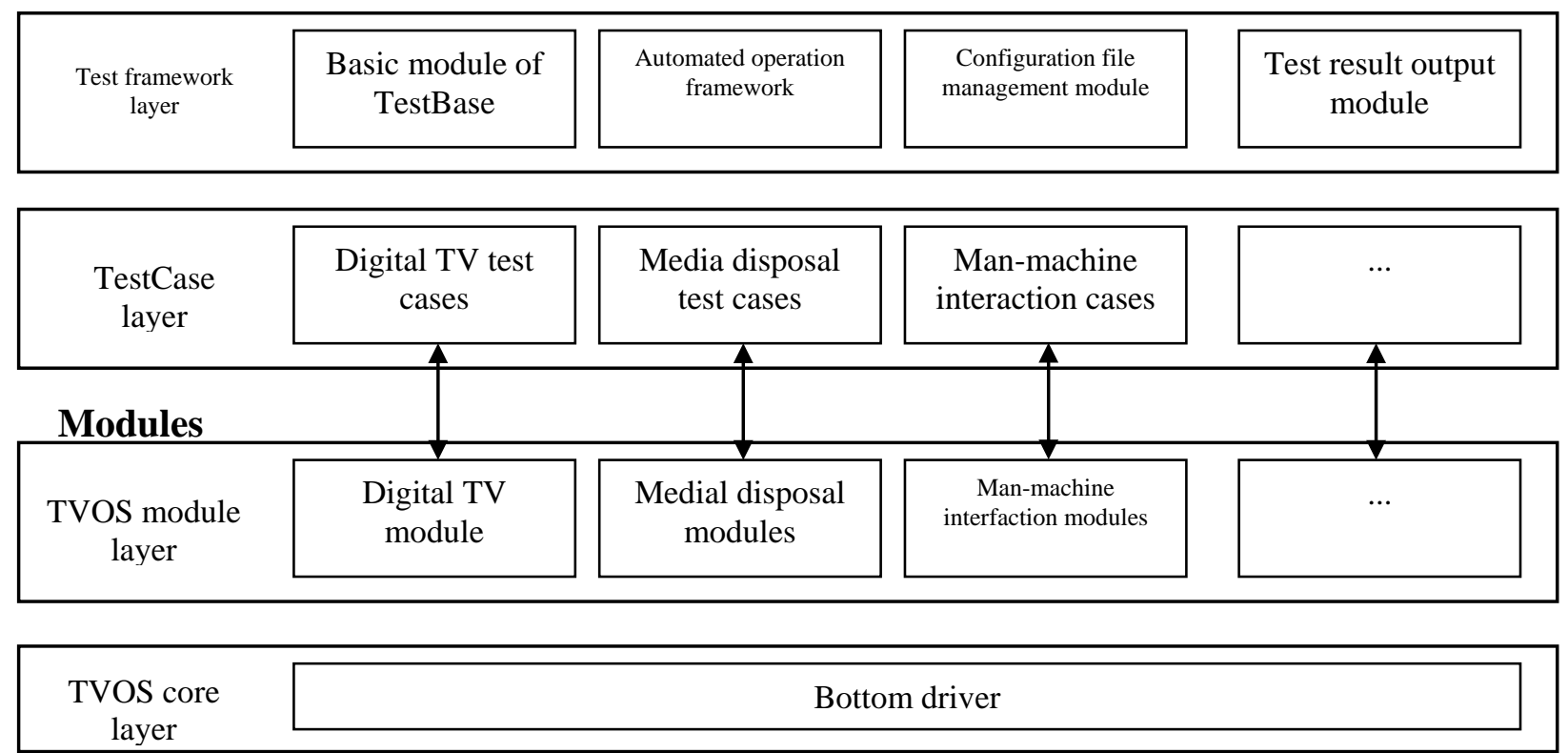

Fig. 1 Automated Test Software Framework of TVOS Modular Layer

\section{Test Process and Strategies}

The testing process and strategy of smart TVOS are shown in Figure 2.

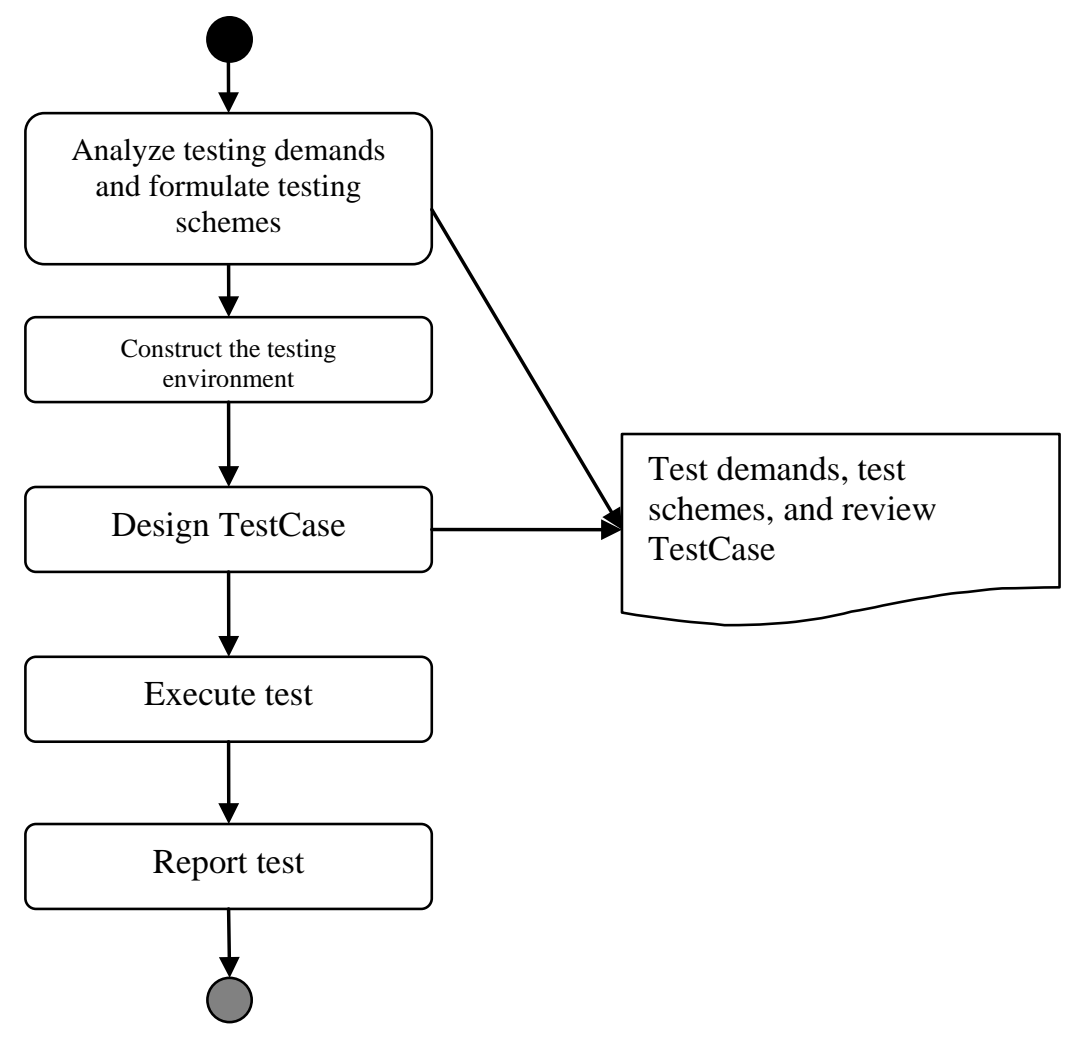

Fig.2 Testing Process of NGB Smart TVOS System

Testing process and strategies: According to the TVOS testing demands, it is necessary to construct the testing environment, design TestCase and ensure that each demand item has the corresponding case. For the primary process of the system, it must be covered. The required TestCase should be confirmed. In addition, it must ensure the entry conditions and expected output, ensure testing environment for executing TestCase, and ensure coverage of testing items. In addition to the 
correctness of the basic points and main process, it also includes system disposal ability when input item is the invalid equivalent item and whether the system gives the correct reminder when output item is the boundary value. The development of testing tools and TestCase should analyze the interface to be tested from the testing perspective, including entry parameters, interface output, and context interface correlation. Based on the testing process and testing strategies, the technical conforming testing of TVOS is realized accurately and efficiently.

\section{The Automated Testing Environment of Smart TV Operating System Based on TVOS}

A. Broadcasting business front end: by displaying the output TS flow of two code players, ASI line is used to connect to two Multiplexers. EPG server outputs EPG information. Then, exchangers are used to send data to the multipliers.

B. Bilateral business front end: this mainly includes store server, network video server, CTS test server, and test set supervisor. The application store server can provide TVOS terminal download, installation and utilization. The network video server provides online video for TVOS terminal to offer VOD. CTS testing server can provide CTS test case set for TVOS to do CTS test. The test set supervisor offers NGB-H and HTML5 test cases for TVOS terminal utilization.

C. Testing equipment: testing equipment is shown in Table 3.

Tab.3 Testing Equipment Listing

\begin{tabular}{|c|c|c|}
\hline No. & Equipment name & Equipment model \\
\hline 1 & TVOS prototype(terminal to be tested) & ZXV10 B720C-A10 \\
\hline 2 & Code flow player s & GT-CP2002 \\
\hline 3 & Code flow analyzers & TV-TS2010 \\
\hline 4 & Multipliers & SMR-010 \\
\hline 5 & Main control client of mupliers & SM-NP1003 \\
\hline 6 & Modulators & HYM-S0020 \\
\hline 7 & DHVP/NTP supervisors & GT-TP1001 \\
\hline 8 & Test set supervisors & TL-SG1024T \\
\hline 9 & Kiomega exchangers & AOC-T2255 \\
\hline 10 & Supervisors & \\
\hline
\end{tabular}

\section{Automated Test Realization of Smart TV Operating System Based on TVOS}

From the lower to the bottom, TVOS can be divided into five layers: Linux core layer, hardware abstract layer, modular layer, executive environment layer and application framework layer, it can realize the overall high-efficient management for the system resources. (1) Linux core layer: this applies the Linux open-source community mainstream version 3.0.31 as the baseline version. Linux core provides the core system service. (2) Hardware abstract layer: this conducts the abstract sealing with the relevant parts in the hardware platform. This is convenient for transplantation. (3) Modular layer: the modular layer realizes the specific functions of TVOS; (4) executive environment layer: Web application environment is the Web browser. (5) Application framework: this applies the programming interface for the exposed functional modules to seal in Java or Web space. The testing system is constructed, configured with the effective tuning parameters of the front-end modulators. Frequency: 650MHz; symbol rate: 6875KSymbol/s, modulation mode: 64QAM; maintain the connection between broadcasting TV signal and internet signal; operate ADB connecting with TVOS terminal to be tested; enter into the automated testing system; operate ./cts-tradefed to execute the following command cts-tf $>$ run cts_-plan tvos-j—disable-reboot, start to test; shown in Figure 3. 


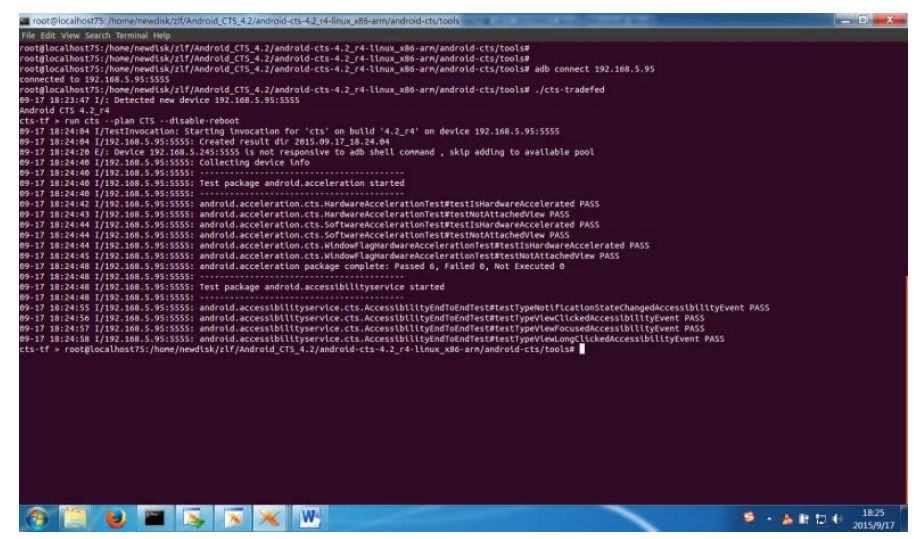

Fig.3 NGB-J Automated Test

\section{Conclusions}

The smart TVOS testing platform and testing method research project combine with actual demands to conduct extensive discussion with multiple manufacturers in the cooperative development group and conduct the in-depth study on the basis of analyzing and studying TVOS framework. The testing suggests that the draft has certain rationality and usability. The later direction should draw a draft in line with the smart TVOS testing platform and testing methods. By using this testing platform, testing tools and testing cases, the technical conforming testing work of TVOS is rapidly conducted.

\section{References}

[1] Zhou Wenpeng, Guan Quan and Lan Jie, The Global Patent Analysis of the Smart TVOS[J], TV Technology, 2016, 02:8-12;

[2] Sheng Zhifan, the Smart TVOS2.0 and Broadcasting Terminal Intelligence[J], Broadcasting and TV Technology, 2016,03:34-39;

[3] Cui Kai, Zhou Kuanjiu, Liang Haoran and Pan Jie, the Generation Methods and Application Study on Android Smart TV TestCase[J], Computer Engineering and Science, 2016, 08:1669-1675;

[4] Liu Chunmei, Han Rui and Guo Zhichuan, the Realization of Automated Testing for the Smart TVOS Modular Layer[J], Network New Media Technology, 2016,05:45-50

[5] Sheng Zhifan, Smart TVOS2.0 — the Main Engine of Broadcasting Terminal Intelligence[J], Audio Visual Circle(Broadcasting Technology), 2016,06:47-58 